\title{
Preserving Privacy in the Internet of Connected Vehicles
}

\author{
Soheila Ghane*, Alireza Jolfaei ${ }^{\dagger}$, Lars Kulik* $^{*}$ Kotagiri Ramamohanarao*, and Deepak Puthal ${ }^{\ddagger}$ \\ ${ }^{*}$ School of Computing and Information Systems, University of Melbourne, Australia, Email: soheila.ghane@ student.unimelb.edu.au \\ ${ }^{\dagger}$ Department of Computing, Macquarie University, Australia, Email: alireza.jolfaei@mq.edu.au \\ $\ddagger$ School of Computing, Newcastle University, UK, Email: deepak.puthal@ newcastle.ac.uk
}

\begin{abstract}
Today's vehicles are advancing from stand-alone transportation means to vehicle-to-vehicle, and vehicle-toinfrastructure communications enabled devices which are able to exchange data through the transportation communication infrastructure. As the IoT and data remain intrinsically linked together, the fast-changing mobility landscape of intent-based networking for the Internet of connected vehicles comes with a great risk of data security and privacy violations. This paper considers the privacy issues in the distributed edge computing, in which the data is communicated between a number of vehicles in the IoT layer and potentially untrusted edge controllers at the edge of the network. The sensory data communicated by the vehicles contain sensitive information, such as location and speed, which could violate the users' privacy if they are leaked with no perturbation. Recent studies suggest mechanisms for randomizing the stream of data to ensure individuals' privacy. Although the past works on differential privacy provide a strong privacy guarantee, they are limited to applications where communication parties are trusted and/or there is no correlation between the users or the featured of sensory data. In this paper, we address this gap by proposing a differentially private data streaming system that adds a correlated noise in the vehicle's side (IoT layer) rather than the transportation infrastructure. Also, our system is able to ensure a strong privacy level over time. The proposed mechanism is data-adaptive and scales the noise with respect to the data correlation. Our extensive experiments demonstrate that the utility of the output generated by our method outperforms the recent approaches.
\end{abstract}

Index Terms-Differential privacy, edge computing, intelligent transportation system, intent-based networking, Internet of connected vehicles.

\section{INTRODUCTION}

The Intelligent Transportation System (ITS) is approaching a level of maturity that gives confidence to many nations around the world to better control and manage the traffic flow across the cities. The ITS has a hierarchical structure with three layers [1], [2]. The bottom layer includes sensors/actuators (IoT devices), such as the mass airflow sensor, the engine speed sensor, oxygen sensor, manifold absolute pressure sensor, and voltage sensor. The IoT devices are subscribed to a Road Side Unit (RSU) or edge controller through Vehicle-to-Infrastructure (V2I) communications. The edge controller monitors road zones and communicates the road rules (speed limit and road toll) and hazards to the vehicles. An edge controller collects the stream of data from the group of subscribed vehicles and communicated required rules. The top layer includes the central traffic control system or cloud server that collects data from vehicles across all spatial regions and provides longer-term data storage and deep analytics.

The Intent-Based Networking (IBN) is a recent example of analyzing such data for the deployment of end-to-end network services in ITS. The IBN incorporates artificial intelligence and machine learning techniques into system administration [3] [4]. It continuously collects data about ITS components including vehicles and edge controllers to learn and optimize the performance and user experience. The continuous collection and aggregation of data from subscribed vehicles enable an edge controller to discover knowledge about the state of edge network and optimize system operations. However, sending the spatio-temporal data of users to an untrusted edge controller exposes the users' privacy to extreme vulnerabilities [5]. Recent studies showed that an untrusted controller is able to uniquely identify a user by a few spatio-temporal data points [6] [7]. The sensitivity of sensory data indicates the necessity of developing privacy-preserving systems such that before the data is received by the edge controller, it is perturbed in the IoT layer (vehicles) to ensure privacy [8].

Developing such a privacy-preserving system for an online streaming application such as ITS is non-trivial. Continuously aggregating the statistics from vehicles leaks more and more information about the users over time. Additionally, the potential correlation between the vehicles or the features in the sensory data increases the chance of revealing sensitive information. Lastly, the evolving nature of ITS requires the proposed system to be highly efficient in computations. Vehicles move rapidly, and they may get subscribed to different edge controllers at different time windows. They can join or leave a group rapidly and hence, the size of vehicular groups, the number of vehicles in edge controller zones, may vary rapidly from time to time. Such variations in the data communication should be handled efficiently. For example, during the traffic congestion, data communication will be maximum while during the off-peak hours, the changes in connectivity may be minimal.

Given the limited computational resources in the vehicles and the evolving nature of ITS over time, a privacy-preserving system is needed such that it provides a strong privacy guarantee over time considering the evolving nature of ITS, is computationally efficient to be implemented in the vehicle's 
side, and ensures high utility of data stream sent to the edge controller.

To address the privacy concern in ITS, we propose a Differentially Private Data Streaming (DPDS) system. DPDS is designed for a distributed edge computing system, where the data from the IoT layer is highly correlated, and IoT devices can quickly change their subscription (join or leave) from one edge controller to another. DPDS employs differential privacy [9] to ensure a strong guarantee on the privacy of users participating in such a dynamic setting. In particular, while many vehicles in an ITS may join or leave the system, DPDS is able to compute an accurate aggregation of data efficiently and ensures that communicating with untrusted edge controllers will not put the privacy of users at risk.

Our contributions. In this paper, we address the existing privacy concerns when vehicles use an untrusted transportation infrastructure, particularly in scenarios where roads are congested with many vehicles which can quickly join or leave different groups and/or traffic zones. The main contribution of our work is a Differentially Private Data Streaming (DPDS) system, which efficiently aggregates the streaming data from vehicles by adding noise in the IoT layer (vehicles). The proposed method ensures a high utility of aggregated data by taking into account the potential correlation in data while adding noise. It also maintains a high level of privacy guarantee, given the evolving nature of data over time. Our extensive experiment confirms the efficiency and utility of DPDS.

\section{RELATED WORK}

Due to the strong theoretical guarantee on the privacy of users, differential privacy has received much attention in data collection [10] [11] and real-time data streaming [12] [13]. However, the proposed systems are based on a trusted server, which severely limits their application [14] [15]. In [12], Fan et al. proposed an adaptive system to release aggregated statistics of real-time, spatio-temporal data under differential privacy. The introduced system uses sampling and filtering techniques to mitigate the effect of added noise over time. Although the mechanism optimizes the allocation of the budget at each timestamp, it is limited to finite data streams. A subsequent work [13] extended the mechanism by considering a sliding window of $w$ time stamps ( $w$-event privacy) and optimizing the budget allocation within the window. Using windows of recent observations enables the mechanism to provide differential privacy on infinite data streams. Although these works provide a good insight into publishing data streams under differential privacy guarantee, they rely on a trusted server which does not fit into many real applications.

To address the problem of untrusted server, a number of works investigated local differential privacy [16] [17]. The intuition of local differential privacy is to randomize each single sensed values in the IoT device before leaving the user's device. The proposed mechanisms aim to improve the utility of aggregated data in the server-side, where the randomized values from all devices are aggregated. Duchi et al. [16] proposed minmax mechanism for publishing numerical values based on local differential privacy. Wang et al. [17] improved the accuracy of minmax on numerical data and extended it for releasing binary and numerical data. However, the proposed mechanism randomly selects $k$ attributes to be perturbed. Reducing the number of sensory value helps the mechanism to reduce the scale of added noise. However, removing some values makes the mechanism impractical in many applications that need the entire sensory data at each timestamp. For example, in an ITS, each vehicle provides a vector of sensed values, and all the values are required for analyzing the traffic data in the edge controller and later in the cloud server [11] [18]. In addition, the proposed mechanisms based on local differential privacy fail to address potential correlation in data. If there is a correlation between the IoT devices or the sensed values in each device are correlated (for example, speed and location values in a transportation application) the above mechanisms are susceptible to the privacy breach.

The mentioned mechanisms carefully allocate the privacy budget at each timestamp. However, continuously computing differentially private values over the time accumulates the privacy loss to a large value even in relatively short time periods [19]. Recently, Joseph et al. [19] applied a filtering technique to save the privacy budget in some timestamps. The introduced mechanism assumes the group's users with similar behaviour are known. Given the groups, the proposed technique spends local privacy budget only if the distribution of a partition has changed significantly. However, the mechanism is limited to binary values. In addition, the budget allocation in the mechanism is uniformly based on the length of the stream, which limits the algorithm to the finite streams.

The limitations in existing mechanisms motivated us to propose our system for numerical values in a setting that the server is not trusted. In an ITS, the server can be a cloud server or an edge controller in which the data is analysed and collected from the vehicles on the road. For brevity, we consider the edge controller as the server since it is the first layer collecting the sensory data from the vehicles.

\section{PReliminaries}

\section{A. Problem Setup and System Model}

We consider a three-layer ITS architecture (Figure 1), where the bottom layer consists of a multitude of IoT devices or vehicles, the middle layer contains untrusted edge controllers and the top layer includes cloud data centers. The bottom layer includes heterogeneous, resource-constrained vehicles, including environmental sensors, cameras, noise monitors, and lighting controls. The middle layer includes edge controllers, which are subscribed to a group of IoT devices. Edge controllers continuously aggregate a stream of data from vehicles and are able to analyze the aggregated data to discover new knowledge about the current state of the edge network with the goal of optimizing system operations. The top layer acts as a global controller and can support heavy duty operations, such as longer-term storage of raw and processed data streams, and deep data analysis. 


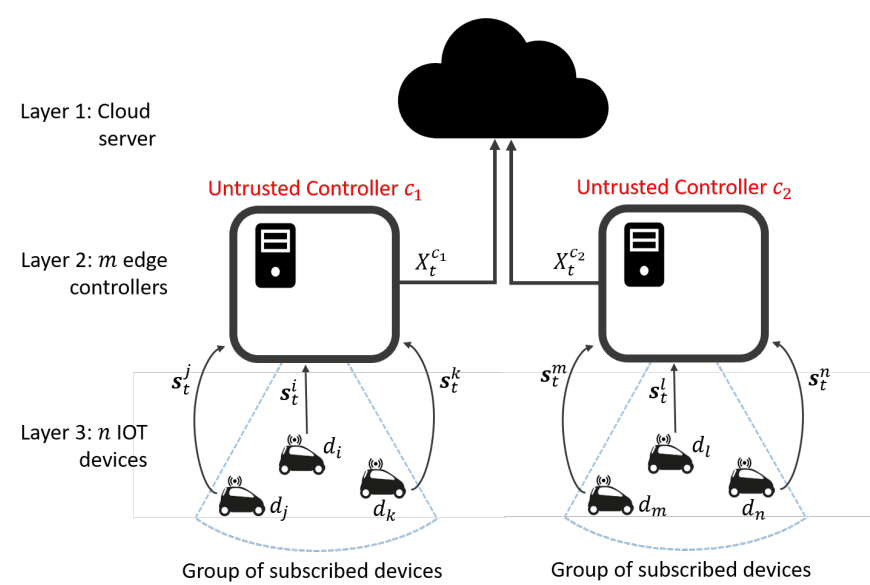

Fig. 1: ITS Architecture: Edge controllers collect data from vehicles and send it to the cloud server.

Let us denote the set of IoT devices and the set of edge controllers by $\mathcal{D}=\left\{d_{1}, d_{2}, \ldots, d_{n}\right\}$ and $\mathcal{C}=\left\{c_{1}, c_{2}, \ldots\right.$, $\left.c_{m}\right\}$, respectively. In our problem setup, IoT devices are able to subscribe to different edge controllers in different timestamps. However, to maintain the system operation, each IoT device needs to be subscribed to only one edge controller in each timestamp. Thus, in each timestamp, there is a group $G \subseteq \mathcal{D}$ of devices subscribed to a controller. In each time instance $t$, an IoT device $d_{i} \in G$ senses a set of features $\boldsymbol{s}_{t}^{i}=\left[f_{i 1}, \ldots, f_{i k}\right]$, where $f_{i j}$ represents the value of $j^{t h}$ feature. We consider a feature as a condition querying the status of IoT device. The value of feature is then a count which represents how the device satisfies the condition, that is, $f_{j} \in[0,1]$.

The IoT device $d_{i} \in G$ sends the sensed data $s_{t}$ to the subscribed edge controller $c$ where the sensory data from all devices in $G$ are aggregated in the form of a two-dimensional matrix as

$$
X_{t}=\left[\begin{array}{cccc}
f_{11} & f_{12} & \ldots & f_{1 k} \\
f_{21} & f_{22} & \ldots & f_{2 k} \\
\vdots & \vdots & \ddots & \vdots \\
f_{n 1} & f_{n 2} & \ldots & f_{n k}
\end{array}\right],
$$

where each row represents the data $s_{t}^{i}=\left[f_{i 1}, \ldots, f_{i k}\right]$ sensed by device $d_{i}$. The $j$-th sensed feature by device $d_{i}$ is denoted by $f_{i j}$. The edge controller collects the time-stamped data $X_{t}$ in instances $t=t_{1}, t_{2}, \ldots$ and/or sends the data to the cloud server. Figure 2 shows the data aggregation in an untrusted edge controller. The following example describes the application of this setting in an ITS.

Example III.1. An ITS collects data from vehicles across a city. Each vehicle represents an IoT device with a unique MAC address, and it is subscribed to the closest traffic controller $c$. In each timestamp $t$, a vehicle $d$ senses data $s_{t}$, that is, about its status and environment, and sends the data stream to the controller through a wireless connection. The communications are typically based on IEEE 1609 and

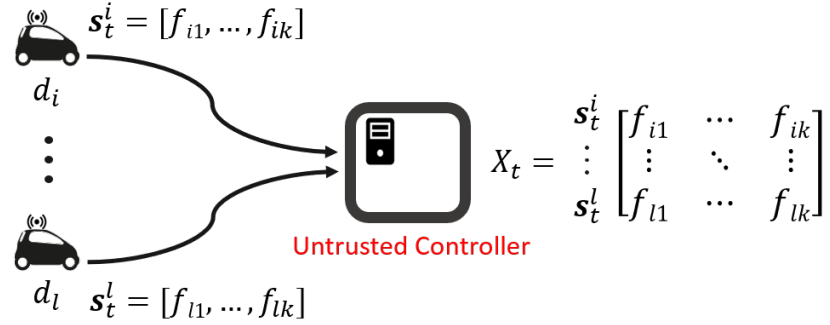

Fig. 2: Data aggregation in an untrusted edge controller.

IEEE 802.11 standards, specifically designed for vehicular communications. The sensed features are, for example, the vehicle's speed, direction, and location. As vehicles move along the roads, they may unsubscribe from one controller and subscribe to a new one. However, the total number of vehicles and traffic controllers are fixed in the city. Total traffic data is then sent to the central traffic management server for further analysis and control.

\section{B. Privacy Definition}

Let us assume that an edge controller $c$ aggregates the timestamped data from the vehicles $\mathcal{D}=\left\{d_{1}, d_{2}, \ldots, d_{n}\right\}$, in the form of $X_{t}$ in instances $t \in\left\{t_{1}, t_{2}, \ldots\right\}$. Since a vehicle represents the driver's behaviour, the sensory data in $X_{t}$ may reveal sensitive information about the system users. The privacy guarantee should ensure the privacy of users involved in $X_{t}$ when the edge controller and the cloud server are not trusted entities. Consider the ITS example (Example III.1). Vehicles move with the speed limit in designated speed zones within certain times, for instance, $40 \mathrm{~km} / \mathrm{h}$ zones during school times. When the edge controller knows the speed of a car is greater than $40 \mathrm{~km} / \mathrm{h}$ during school times, the car might be subject to fine. Additionally, knowing that an individual has been in a particular location in a specific time, may reveal sensitive information about that individual's habits or interests. In other words, the time-stamped feature values of individuals are sensitive, and the privacy goal is to protect them.

Given the data matrix $X_{t}$, a matrix $X_{t}^{\prime}$ is considered as a neighbor if the two matrices differ in a single user that is represented by a row [9]. For data matrices in a data stream, we define $\epsilon$-differential privacy as follows:

Definition III.1. (Differential privacy) A randomized mechanism $\mathcal{M}$ gives an $\epsilon$-differential privacy if for all neighboring data matrices $X_{t}$ and $X_{t}^{\prime}$ and possible outputs $O \subseteq$ Range $(M)$,

$$
\operatorname{Pr}\left[\mathcal{M}\left(X_{t}\right) \in O\right] \leq e^{\epsilon} \times \operatorname{Pr}\left[\mathcal{M}\left(X_{t}^{\prime}\right) \in O\right],
$$

where probability is over the randomness of $\mathcal{M}$.

The parameter $\epsilon$, named privacy budget, represents the level of privacy. A lower privacy budget depicts a stronger privacy guarantee, and a larger value shows a higher risk of a privacy breach. Accordingly, differential privacy defines two composition properties for constructing complex mechanisms: 
sequential and parallel compositions. Given $k$ mechanisms $\mathcal{M}_{1}, \ldots, \mathcal{M}_{k}$, where $\mathcal{M}_{i}$ satisfies $\epsilon_{i}$-differential privacy for $i(1 \leq i \leq k)$, sequential and parallel compositions are as follows:

- Sequential composition: The execution of $\mathcal{M}_{1}\left(X_{t}\right), \ldots$, $\mathcal{M}_{k}\left(X_{t+w}\right)$ satisfies $\left(\sum \epsilon_{i}\right)$-differential privacy. In other words, applying mechanisms on data matrices of sequential timestamps in a data stream accumulates the privacy budget and in turn degrades the privacy guarantee.

- Parallel composition: Given $k$ disjoint controllers $c_{1}, \ldots$, $c_{k}$, the parallel execution of $\mathcal{M}_{1}, \ldots, \mathcal{M}_{k}$ on data matrices $X_{t}^{c_{1}}, \ldots, X_{t}^{c_{k}}$ satisfies $\max \left\{\epsilon_{i}\right\}$-differential privacy.

The composition properties are important in reasoning about the total privacy level of a mechanism. However, a naive approach in composing mechanisms may result in a very low privacy level due to the accumulation of privacy budgets. In particular, the evolving nature of a stream data over time requires a mechanism that carefully uses the privacy budget. We will describe how our proposed mechanism saves the privacy budget only for timestamps that new information is provided by the IoT devices. Our mechanism can be interpreted as an instance of $w$-event $\epsilon$-differential privacy [20]. We first define $w$-neighboring on a set of data $X=\left\{X_{1}, X_{2}, \ldots\right.$, $\left.X_{t}\right\}$.

Definition III.2. (w-neighboring [20]) Given a positive integer time stamp $w$, the two sets of data $X_{(1, t)}=\left\{X_{1}, X_{2}\right.$, $\left.\ldots, X_{t}\right\}$ and $X_{(1, t)}^{\prime}=\left\{X_{1}^{\prime}, X_{2}^{\prime}, \ldots, X_{t}^{\prime}\right\}$ are $w$-neighboring if

1) for $i(1 \leq i \leq t), X_{(1, t)}[i] \neq X_{(1, t)}^{\prime}[i]$, it holds that $X_{(1, t)}[i]$ and $X_{(1, t)}^{\prime}[i]$ are neighbors.

2) for each $X_{(1, t)}[i], X_{(1, t)}[j], X_{(1, t)}^{\prime}[i], X_{(1, t)}^{\prime}[j]$ with $i<j$, $X_{(1, t)}[i] \neq X_{(1, t)}^{\prime}[j]$ and $X_{(1, t)}[i] \neq X_{(1, t)}^{\prime}[j]$, it holds that $j-i+1 \leq w$.

The $w$-neighboring intuitively describes two streams $X_{(1, t)}$ and $X_{(1, t)}^{\prime}$ are neighbor when (1) their elements are pairwise the same or neighboring, and (2) all their neighboring streams can fit in a window of up to $w$ time stamps.

Definition III.3. ( $w$-event $\epsilon$-differential privacy) Let $\mathcal{M}$ be a randomized mechanism that takes a set of data $X_{(1, t)}=$ $\left\{X_{1}, X_{2}, \ldots, X_{t}\right\}$ as input and generates an output $O \subset$ Rage $(\mathcal{M}) . \mathcal{M}$ satisfies $w$-event $\epsilon$-differential privacy if for all $w$-neighboring streams $X_{(1, t)}$ and $X_{(1, t)}^{\prime}$, for all time stamps $i(1 \leq i \leq t)$ and $O \subset$ Range $(f)$ it holds that

$$
\operatorname{Pr}\left(\mathcal{M}\left(X_{i}\right)=O\right) \leq \exp (\epsilon) \times \operatorname{Pr}\left(\mathcal{M}\left(X_{i}^{\prime}\right)=S^{*}\right) .
$$

This privacy model provides $\epsilon$-differential privacy on data streams and our mechanism improves the privacy level by applying an effective filtering strategy as explained in Section IV-C.

Depending on the analysis tasks, the data matrix $X_{t}$ in a data stream may be used for different functions. For example, given the traffic data in Example III.1, some functions can be $Q_{1}$ : "the number of vehicles crossing the street $x$ at time step $t$ "; $Q_{2}$ : "the number of vehicles crossing the street $x$ at time window $w$ " where $w=\left\{t_{0}, t_{1}, \ldots, t_{w}\right\}$; or $Q_{3}$ : "whether the number of vehicles crossing the street $x$ at time window $w$ is greater than a threshold $\delta$ ".

Given a function $Q$, the standard mechanisms to achieve differential privacy is to add random noise to the output of $Q$ [9]. The noise is carefully calibrated by the sensitivity of the function, that is, maximum changes in the function output by adding or removing a user (a row in data matrix $X_{t}$ ).

Definition III.4. (Sensitivity) Given a function $Q: X_{t} \rightarrow \mathbb{R}^{n}$ and any two neighbor data matrices $X_{t}$ and $X_{t}^{\prime}$, the sensitivity of $Q$ is

$$
\Delta_{\max } Q=\left\|Q\left(X_{t}\right)-Q\left(X_{t}^{\prime}\right)\right\|_{1}
$$

In our setting, we have $Q \in[0,1]$ meaning that adding or removing a feature from a user's data would affect the query at most by 1 . However, a user may have sensed multiple features and hence, the vector $s$ in Figure 2 is $|s|>1$. This results in $\Delta Q>1$.

The Laplace mechanism [9] is a well-known approach in achieving $\epsilon$-differential privacy. The mechanism adds an independent noise from a Laplace distribution to the output of $Q$ where the noise scale is calibrated for the sensitivity of $Q$. We use $\operatorname{Lap}(\sigma)$ to denote a Laplace distribution with scale $\sigma$ and mean 0 .

Definition III.5. (Laplace mechanism) Given a data matrix $X_{t}$ and a function $Q: X_{t} \rightarrow \mathbb{R}^{n}$, the Laplace mechanism denoted by $\operatorname{LM}\left(X_{t}, Q\right)$ is defined as

$$
L M\left(X_{t}, Q\right)=Q\left(X_{t}\right)+\operatorname{Lap}\left(\Delta Q \epsilon^{-1}\right)^{n} .
$$

In general, using a plain Laplace mechanism on data streams may cause privacy breach. In real applications, there might be correlations between the entries in the data matrix (correlation between rows or columns). Correlation in data enables an adversary to reduce the privacy guarantee and infer sensitive information of users. The Laplace mechanism does not consider the correlations. In addition, the evolving nature of data streams enables the adversary to compute an accurate estimate of the sensitive information by aggregating the noisy values over time.

Example III.2. Following Example III.1, a group of vehicles in a road, driving close together, would move with a similar speed. Also, it is expected that vehicles move within the speed limit in designated speed zones within certain times. The location and speed features are strongly correlated. This correlation can leak identifiable information of each vehicle by answering the query functions over time.

A principled mechanism is required to consider both the correlation and the evolving nature of data streams in adding noise for ensuring the differential privacy.

\section{OUR DIFFERENTIALLY PRIVATE SYSTEM}

This section describes our Differentially Private Data Stream (DPDS) system for privately transferring data streams in a distributed setting when the server is untrusted (explained 


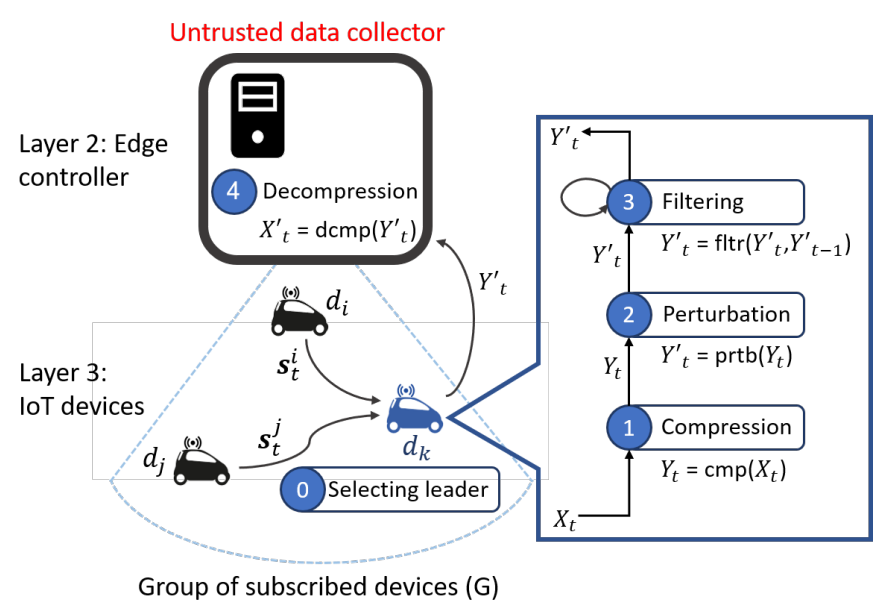

Fig. 3: DPDS mechanism.

in Section III-A). Figure 3 shows an overview of DPDS in an ITS. In the DPDS system, the vehicles do not directly send the sensitive data $s_{t}$ to the untrusted edge controller. In our proposed system, the group of subscribed vehicles to the controller, namely group $G$, select a group leader in step (0) and send the data to the leader (depicted by blue) to ensure privacy before transferring the data to the controller. The group leader, collects $s_{t}^{i}$ from every device (vehicle) $d_{i} \in G$ and makes a data matrix $X_{t}$ to be perturbed under differential privacy. The group leader then computes the perturbed data $Y_{t}^{\prime}$ through three steps: (1) compression, (2) perturbation, and (3) filtering. The untrusted controller receives the perturbed data $Y_{t}^{\prime}$ and decompresses it to achieve $X_{t}^{\prime}$, that is, perturbed data in the original space. Postponing the decompression to the controller improves the efficiency of the system due to its computational power comparing to an IoT device. Studying the behaviour of subscribed vehicles requires querying over all collected data in $X_{t}$ to count the number of devices with specific behaviour. For example, we may be interested in the number of vehicles driving with a particular speed and in a specific direction. Given a data matrix $X_{t}$, such counting task are named count query over $X_{t}$. The count query is a principal query type in most analysis applications, and in this paper, we consider it as the base analysis function.

In our proposed system, instead of perturbing the data directly by adding noise to the entries of $X_{t}$, we first compress the data and then add noise to ensure privacy. The compression enables DPDS to improve the utility of output by: (1) breaking potential dependencies between the entries of $X_{t}$; and (2) pruning insignificant entries in data with no privacy cost. DPDS uses a total privacy budget $\epsilon$ at each timestamp $t$ to perturb the data and ensures $\epsilon$-differential privacy. Since the privacy budget is limited, the filtering step manages the usage of the budget to maintain the longevity of privacy and utility due to the sequential composition theorem. Algorithm 1 describes computational steps of DPDS system: Compression, Perturbation and Filtering. In the following, we describe the steps of the DPDS system in details.

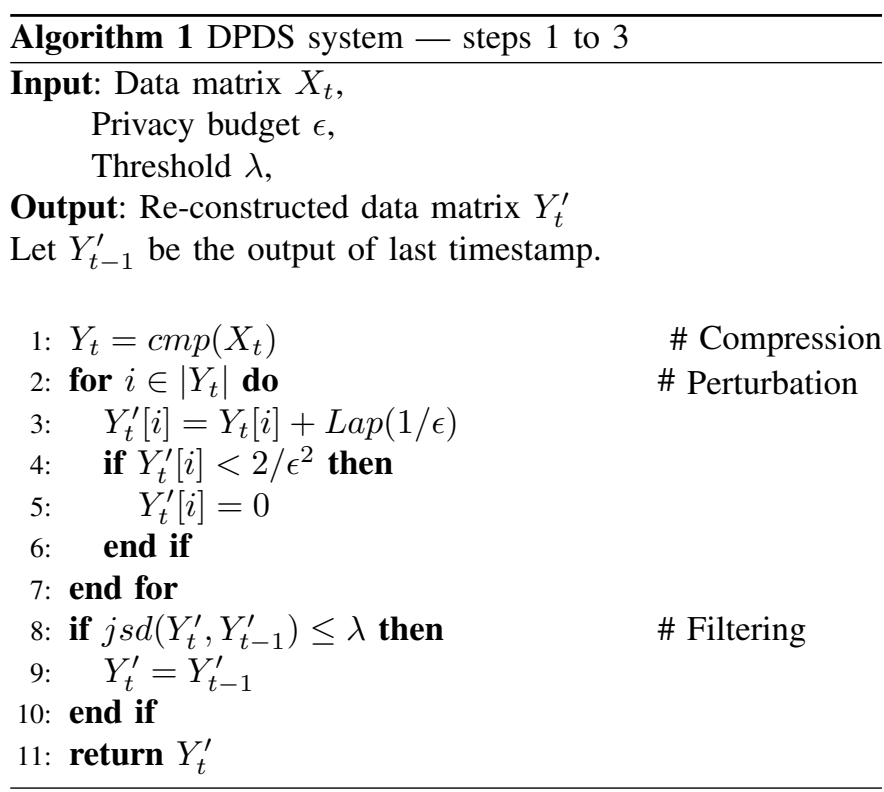

\section{A. Compression}

The vehicles subscribed to the edge controllers, that is, rows in $X_{t}$, and also, the features sensed by each vehicle, that is, columns in $X_{t}$, can be correlated. For instance, the vehicles driving in a road often move with similar speeds, particularly, in speed zones which may vary in different time windows in a day. The location of a vehicle and its corresponding speed are correlated features which can leak identifiable information. A similar correlation can be defined between IoT devices.

The correlation between entries of the data matrix $X_{t}$ can force the addition of a large scale noise to the data in order to ensure differential privacy. Any orthogonal transformation on rows and columns of $X_{t}$ can transfer the data to a space where the entries are independent. Such a transformation allows adding independent noise to each entries of $X_{t}$ and hence, improves the utility of the output. However, in online streaming, such transformation should be applied to the collected data $X_{t}$ at each timestamp $t$. Thus, an efficient approach should be used for the orthogonal transformation. For example, if the transformation is data-dependent, the matrix of transformation should be computed based on the collected data at each timestamp and this can impose excessive computational overheads on vehicles and the system.

In DPDS, we make use of a Haar Wavelet transform [21]. The Haar transformation is a well-known, efficient, orthogonal transformation and it is widely used for compression of data [22] [23]. Given a data matrix $X_{t}$, the Haar transformation and compression work as follows:

1) Transformation: It transforms $X_{t}$ into a new matrix $Y_{t}$, which consists of (i) an approximation of data, namely $Y_{t}^{a}$, and (ii) wavelet coefficients, namely $Y_{t}^{c}$. Figure 4 shows an example of a Haar transformation on an image, that is a matrix of data.

2) Thresholding: If any value in $Y_{t}$ is less than a threshold $\theta$, it would suppress the value to 0 .

3) Inverse transformation: This transformation reconstructs 


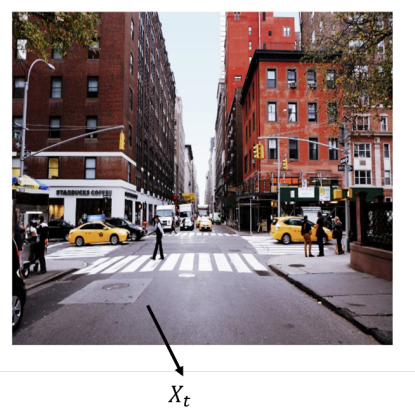

(a)

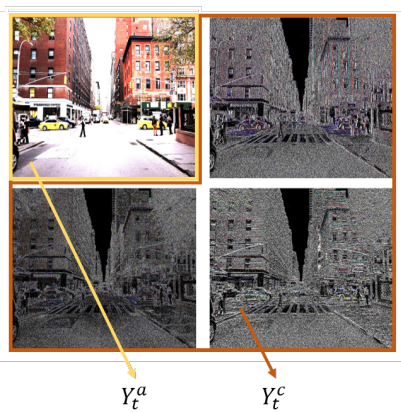

(b)
Fig. 4: Haar transformation example: (a) an image as a 2D matrix of data $X_{t}$; (b) the transformed data $Y_{t}$ after a one-level Haar transformation consisting of data approximation $Y_{t}^{a}$ and wavelet coefficients $Y_{t}^{c}$.

the data matrix $Y_{t}$ using the modified approximation $Y_{t}^{a}$ and the coefficients $Y_{t}^{c}$.

In the DPDS system, the Haar transformation is performed in the compression phase, and the perturbation phase applies the Haar thresholding. The inverse transformation step is postponed to the controller side because the transformation matrix is known and also it reduces the computational overhead of IoT layer (vehicles). In the following, we describe the Haar transformation in DPDS. The matrix of transformation using a one level Haar wavelet is

$$
H_{2}=\left[\begin{array}{cc}
1 & 1 \\
1 & -1
\end{array}\right] .
$$

The size of the Haar matrix is defined based on the data size and it follows the above structure. For example, given a data matrix $X_{t}$ with size $4 \times 4$, the Haar matrix would be

$$
H_{2}=\left[\begin{array}{cccc}
1 & 1 & 0 & 0 \\
1 & -1 & 0 & 0 \\
0 & 0 & 1 & 1 \\
0 & 0 & 1 & -1
\end{array}\right] .
$$

The Haar matrix is applied to rows and then the columns of $X_{t}$ in the Compression phase of DPDS. As shown in [24], the computation complexity of the Haar transform is $O(d \log d)$, where $d$ is the number of entries in $X_{t}$. Given the logarithmic complexity of the Haar transform implementation, the group leader and later the edge controller in our system are able to efficiently apply the transformation and inverse transformation of Haar wavelet, respectively. Note the Haar transform requires an even number of rows and columns in data matrix. To satisfy this requirement, we amend the data by adding random rows or columns.

Let $\operatorname{cmp}(\cdot)$ denote a function that transforms a data matrix using a Haar wavelet transform. In other words,

$$
Y_{t}=c m p\left(X_{t}\right) \text {, }
$$

where $X_{t}$ is the input data matrix with size $d$ and $Y_{t}$ is the output data matrix with size $d . Y_{t}$ contains the approximation values $Y_{t}^{a}$ and the wavelet coefficients $Y_{t}^{c}$. The entries of $Y_{t}$ are independent and ready to be perturbed in the next step of the DPDS system.

\section{B. Perturbation}

Given the transformed data matrix $Y_{t}$, with independent entries, in this step, DPDS perturbs the data as $Y_{t}^{\prime}$ to ensure differential privacy. The Laplace mechanism [9] is used to add noise to $Y_{t}$. As the entries of $Y_{t}$ are independent, adding a random value driven from the Laplace noise to each entry provides an $\epsilon$-differential privacy. Given that $d$ is the number of entries in $Y_{t}$,

$$
Y_{t}^{\prime}=Y_{t}+\operatorname{Lap}(\sigma)^{d}
$$

where $\sigma=\Delta Q / \epsilon$ is the variance of the Laplace noise (see Section III-B).

The added noise should be scaled to fit the sensitivity of function $Q$. Recall that the concept of sensitivity captures the maximum changes in the output caused by presence or absence of a vehicle. As defined in Section III-A, each sensed feature in data $f \in X_{t}$ maintains a value such that $f \in[0$, $1]$ and a vehicle may sense multiple features $\left\{f_{1}, \ldots, f_{m}\right\}$ at each timestamp. Thus, the presence or absence a vehicle may change the total values in $X_{t}$ by at most $\sum f_{j}, \forall j \in\left\{f_{1}\right.$, $\left.\ldots, f_{m}\right\}$. This means $\Delta Q=\sum_{j=1}^{m} f_{j} \geq 1$ which results in a large scale noise.

To reduce the scale of added noise, we normalize the original data $X_{t}$ for each vehicle, that is, a row in $X_{t}$. With our normalization, the maximum change by the presence or absence of a device would be 1 . Given the independent entries in $Y_{t}$, the normalization ensures $\Delta Q=1$ and hence, the variance of noise to be added to each entry of $Y_{t}$ is $\sigma=1 / \epsilon$.

Thresholding: Although the transformation and normalization reduce the scale of added noise, there might be small values in $Y_{t}$ which are highly sensitive to the noise. Especially the wavelet coefficients in $Y_{t}^{c}$ are known to be often small values and adding noise may degrade the utility of data significantly. Hence, after adding Laplace noise to the entries of $Y_{t}$, we determine some entries to be suppressed to 0 . We introduce a thresholding strategy for this suppression based on the signal to noise ratio. We associate the threshold

$$
\theta=\mathbb{E}\left(\operatorname{Lap}(1 / \epsilon)^{2}\right)=2 / \epsilon^{2}
$$

to remove values smaller than the expected value of the added noise. In our thresholding strategy, if a value $v \in Y_{t}^{\prime}$ is less than $\theta$, we modify the value to 0 in $Y_{t}^{\prime}$.

Let $\operatorname{prtb}(\cdot)$ be the function of perturbation. It takes the matrix of coefficients $Y_{t}$ as an input and generates the modified $Y_{t}^{\prime}$ after adding noise and thresholding.

\section{Filtering}

Given $Y_{t}^{\prime}$, the group leader can send the perturbed coefficients to the controllers. Then, in the controller, the perturbed data matrix $X_{t}^{\prime}$ can be computed by applying the inverse transformation on $Y_{t}^{\prime}$ according to the Haar transformation described in the Compression phase. DPDS uses $\epsilon$ privacy budget to compute $Y_{t}^{\prime}$. Since a new $Y_{t}^{\prime}$ should be computed at each timestamp $t$, this causes degrading the level of privacy due to the serial composition theorem (see Section III-B). We 
introduce our filtering technique to save the privacy budget only for significant changes in the streaming data.

Definition IV.1. (Significant change) Let $Y_{t-1}^{\prime}$ be the perturbed observation at timestamp $t-1$. A new perturbed observation $Y_{t}^{\prime}$ at timestamp $t$ is considered as a significant change in the streaming data if we have

$$
j s d\left(Y_{t}^{\prime}, Y_{t-1}^{\prime}\right)>\lambda,
$$

where $j \operatorname{sd}(\cdot)$ is the Jensen Shannon divergence and $\lambda$ is a given threshold.

Intuitively, if the new observation at time $t$ is not significantly different from the previous sensory data at time $t-1$, it does not provide new information. Thus, the system can filter the new observation and use previous data from time $t-1$. Since our filtering uses the perturbed data $Y_{t}^{\prime}$ and $Y_{t-1}^{\prime}$, it does not affect the privacy guarantees. The group leader only needs to maintain $Y_{t}^{\prime}$ at each timestamp for the filtering purpose. Jensen Shannon divergence (JSD) is a method for measuring the similarity between two probability distributions. Considering the dataset at each timestamp as a probability distribution, we use the JSD to compare the distribution datasets at consecutive timestamps. In our experiments, we show that our filtering technique accurately captures informative changes in the data stream and maintains the utility of output.

\section{Selecting group leader}

In the DPDS system, the subscribed vehicles to a controller make a group and send their sensory data to the group leader at each timestamp. We numbered the leader selection as the step zero in DPDS system because it may not happen at each timestamp. The vehicles in a group select a new leader only when it is the first timestamp the group is constructed, or the current leader leaves the group. The new leader receives the last output of the system from the controller to be used in the filtering step.

Many strategies have been introduced for an efficient selection of group leaders in a setting like our system where a set of IoT devices (in our case, vehicles) communicate in a distributed environment [25]. These algorithms select an IoT device such that it optimizes the energy consumption of the system while maintaining the required resources for potential computations as a leader. In addition, potential security attacks are considered to avoid selecting a malicious IoT device as the leader. Any of the existing strategies can be used in our DPDS system.

\section{Formal ANALYSis}

In this section, we show that our proposed system provides a strong privacy guarantee with a high utility. We also analyze the efficiency of computational steps in the DPDS system, that is, Compression, Perturbation and Filtering, as explained in Algorithm 1.

Privacy: We show our system provides an $\epsilon$-differential privacy guarantee.

Theorem V.1. DPDS satisfies $\epsilon$-differential privacy.
Proof. DPDS consists of a one step for selecting the group leader, three main steps for computing the noisy data, and one step in the controller to decompress the noisy data (Figure 3). The group leader selection does not harm the privacy of vehicles as it is done in the IoT layer, and no information about the vehicles is transferred to the edge controller layer. At each timestamp, the Perturbation step uses an $\epsilon$ privacy budget to perturb the data before sending it to the edge controller. The Filtering step reduces to privacy cost to zero at some timestamps by using the noisy data from the previous timestamp. Both Filtering and Decompression steps are applied after adding noise and hence, does not affect the differential privacy guarantees. The Compression step is applied in the IoT layer, which does not leak any information to other layer and hence does not affect privacy. Note that our model can also be interpreted as a $W$-event $\epsilon$-differential privacy [20], where $w$ is a time window in which the system is using the noisy values from previous timestamps and only uses $\epsilon$ to compute noisy values in the first timestamp of the window. The size of the window varies depending on the data dynamics, and the system adaptively decides about it.

Efficiency: Let $d$ be the number of entries in $X_{t}$. Algorithm 1 computes the output $X_{t}^{\prime}$ with $O(d \cdot \log d+d)$ computation complexity. Using a fast Fourier transform [24], the computation complexity of the Compression phase is $O(d \cdot \log d)$. Adding noise and thresholding in the Perturbation phase would take $O(d)$ operations together. Additionally, the computations in Compression and Perturbation phases can be in-place and this makes the DPDS memory consumption more efficient.

Utility: We measure the utility of the output data using the sum of squared errors (SSE) [26].

Theorem V.2. Given the reconstructed data $X_{t}^{\prime}$ in controller, and the set of values $K$ modified under thresholding,

$$
\mathbb{E}\left\|X_{t}-X_{t}^{\prime}\right\|_{2}^{2}=\sum_{v \in K} v+\frac{2(d-|K|)}{\epsilon^{2}}
$$

Proof. The Haar transformation is able to reconstruct the original data without losing information. However, in our DPDS system, adding noise and thresholding deviates the transformed data which may affect the accuracy of the reconstructed data matrix. The error of the system is then due to the added noise and the modified values (suppressed to 0 ).

Since the Haar transformation is an orthonormal transformation, it preserves the $L_{2}$-norm. Hence, $\left\|X_{t}-X_{t}^{\prime}\right\|_{2}=$ $\left\|Y_{t}-Y_{t}^{\prime}\right\|_{2}$, and therefore the sum of square error of $X_{t}^{\prime}$ is $\mathbb{E}\left\|X_{t}-X_{t}^{\prime}\right\|_{2}^{2}=\mathbb{E}\left\|Y_{t}-Y_{t}^{\prime}\right\|_{2}^{2}$. Without thresholding, for each $v^{\prime} \in Y_{t}^{\prime}$, we have $v^{\prime}=v+\operatorname{Lap}(1 / \epsilon)$, where $v$ is its corresponding value in $Y_{t}$. Since $\operatorname{Lap}(1 / \epsilon)$ is independently added to value $v \in Y_{t}$, the square error is $\mathbb{E}\left(\operatorname{Lap}(1 / \epsilon)^{2}\right)=2 / \epsilon^{2}$. Given $K$ as the set of values which are modified to 0 in $Y_{t}^{\prime}$ 


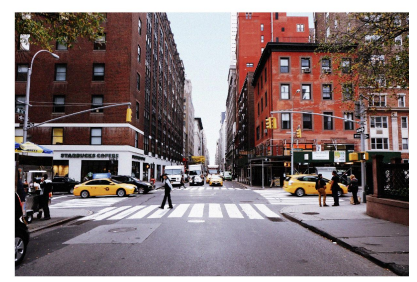

(a) $\epsilon=0.05$

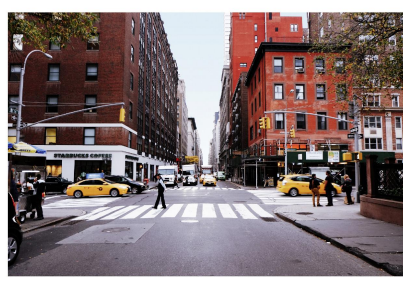

(b) $\epsilon=0.2$

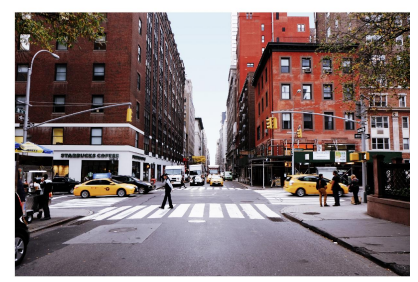

(c) $\epsilon=0.5$

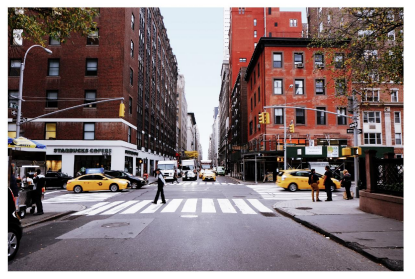

(d) $\epsilon=1.0$

Fig. 5: Utility of the DPDS system.

by thresholding and $d=\left|Y_{t}^{\prime}\right|$, the SSE of $X_{t}^{\prime}$ is

$$
\begin{array}{r}
\mathbb{E}\left\|X_{t}-X_{t}^{\prime}\right\|_{2}^{2}=\mathbb{E}\left\|Y_{t}-Y_{t}^{\prime}\right\|_{2}^{2} \\
=\sum_{v \in K} v+\sum_{v \in Y_{t}^{\prime}, \notin K} \frac{2}{\epsilon^{2}}=\sum_{v \in K} v+\frac{2(d-|K|)}{\epsilon^{2}} .
\end{array}
$$

However, it is known that the wavelet coefficients $Y_{t}^{c}$ are small values and removing them does not significantly affect the utility of the reconstructed data matrix $X_{t}^{\prime}$. As our thresholding strategy aims to remove such small values, the corresponding error is negligible, that is, $\sum_{v \in K} v \approx 0$, and hence,

$$
\mathbb{E}\left\|X_{t}-X_{t}^{\prime}\right\|_{2}^{2}=\mathbb{E}\left\|Y_{t}-Y_{t}^{\prime}\right\|_{2}^{2} \approx \sum_{v \in Y_{t}^{\prime} \notin K} \frac{2}{\epsilon^{2}} .
$$

\section{EVALUATIONS}

In this section, we evaluate the performance of our DPDS system. The evaluation results are presented in two parts:

- The generated output by DPDS system has a high utility. We visualize the utility of our model on a random image, where the transformation technique is inspired from [27].

- The DPDS system is highly scalable and stable compared to the state-of-the-art mechanisms. We show that our system significantly outperforms the competing mechanisms in maintaining the utility of data over time and also in different numbers of vehicles are subscribed to an edge controller. A synthetic dataset is used for these evaluations.

\section{A. Experimental setup}

In the experiments, we set the filtering threshold to $\lambda=0.1$. Considering the JSD as a measure with a range $[0,1]$, this threshold applies a strict filtering strategy to ensure capturing changes in the data stream over time. Unless otherwise specified, we consider a group size of 100 and a run-time of 5 hours in our experiments.

Dataset: We use a simulated dataset provided by TAPASCologne institute [28]. The dataset mimics the car traffic of a large metropolitan area in Cologne, Germany by location and Speed attributes. The data covers a region of 400 square kilometres for a period of 24 hours in a typical working day and comprises more than 700,000 individual car trips. We coarsen the sampling rate to 10 seconds to achieve a uniform sampling rate for the entire data. We constructed Direction and Vehicle Status attributes based on provided features to have more correlated features. The features were then scaled to a range in $[0,1]$. For example, the direction was first quantized into 4 degrees: North, East, South, and West. Then, each direction is mapped to a range in $[0,1]$ interval, that is, North to $[0,0.25)$, East to $[0.25,0.5)$, and etc. In our experiments, we sampled groups of cars with size 25, 50, 100, 200, 400, and 800.

Measure: We use a Mean Absolute Error (MAE) and a Mean Relative Error (MRE) for measuring the utility. Let $X_{t}$ and $X_{t}^{\prime}$, respectively, be the original data and the reconstructed data in the controllers. The MAE and MRE for the two data matrices are [29]

$$
\begin{gathered}
M A E=\frac{1}{\left|X_{t}\right|} \sum_{a \in X_{t}, a^{\prime} \in X_{t}^{\prime}}\left|a-a^{\prime}\right|, \\
M R E=\frac{1}{\left|X_{t}\right|} \sum_{a \in X_{t}, a^{\prime} \in X_{t}^{\prime}} \frac{\left|a-a^{\prime}\right|}{\max (r, a)},
\end{gathered}
$$

where $r=\frac{1}{100} \sum_{a \in X_{t}} a$ to mitigate the effect of excessively small values.

\section{B. Utility evaluation}

Figure 5 shows the utility of our system by modifying the privacy budget. A random image is chosen to describe the performance of our mechanism in adding noise and the effectiveness of the thresholding. The results depict the utility of decompressed data, even in stingy privacy budgets. The reason is that our thresholding strategy mostly targets values that are very small, where if the noisy value was kept, it would degrade the utility of output. Also, it is known that in the Haar transformation, the wavelet coefficients are often significantly smaller than the approximation elements and the effect of removing them is negligible in the utility of reconstructed data. In other words, our thresholding removes elements of data (modifying to 0 ) that have a negligible effect on the utility and also maintaining the noisy value of them would potentially degrade the utility.

\section{Scalability and stability evaluation}

From existing works, Duchi [17], Wang [17] are recent algorithms proposed for a similar setting as our problem. When the server is untrusted, a user senses multiple attributes and the privacy should be ensured in the user's side. However, these mechanisms add noise to the sensory data of each user individually and then aggregate the noisy data in the 

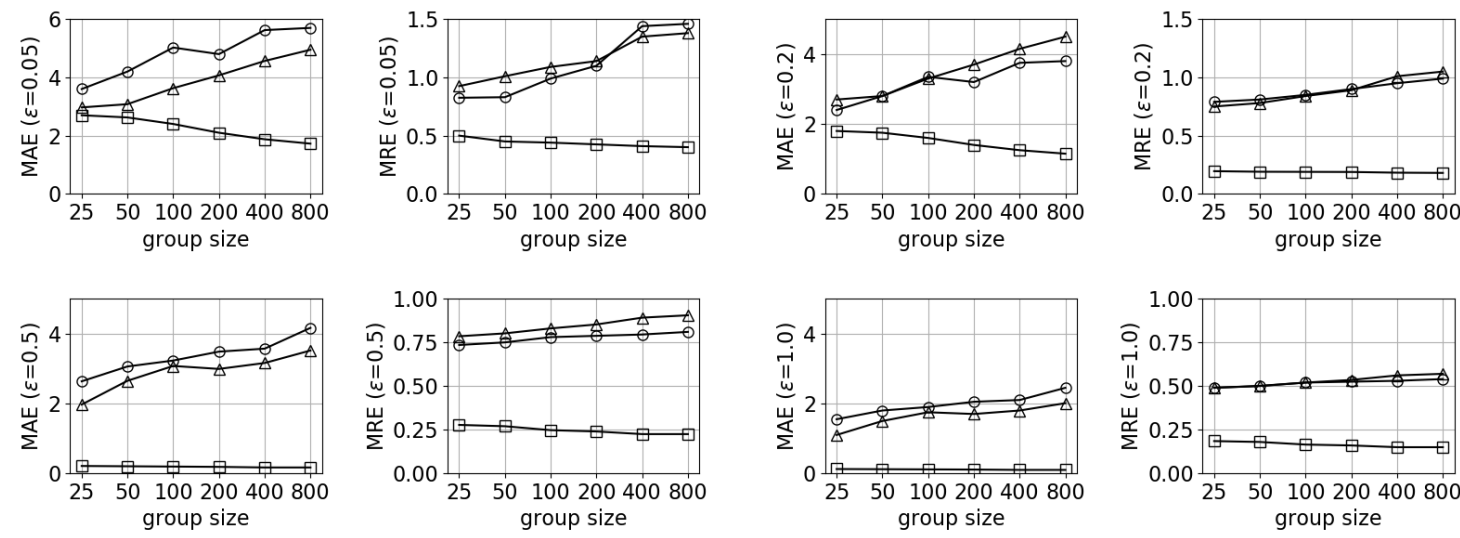

$\square$ dpds $\multimap$ wang $\triangle$ duchi

Fig. 6: Scalability of DPDS versus existing algorithms.
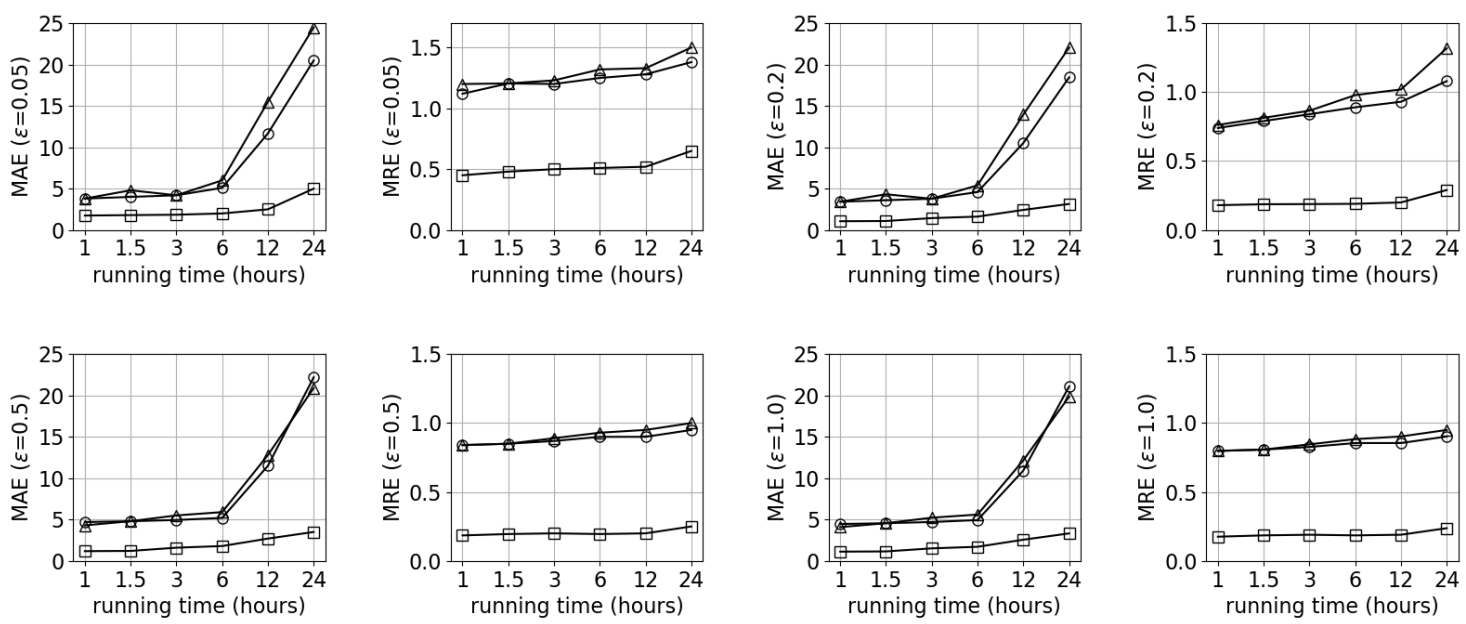

$\square$ dpds $\quad$ wang $\triangle$ duchi

Fig. 7: Stability of DPDS versus existing algorithms.

server-side. Note that some of the existing mechanisms do not consider the potential correlation among the features of data or the users, which may result in a privacy breach. We do not consider this problem in our evaluations. The reported results are the average of 5 independent runnings of the algorithms.

Figure 6 depicts the scalability of algorithms on a different number of vehicles. By scalability, we mean the utility of output should not significantly change by modifying the number of subscribed vehicles in the group. The figures show the scalability of algorithms in a range of privacy budgets. As the results show, DPDS significantly outperforms the other algorithms. The reason is that in DPDS, the noise is calibrated to the sensitivity as well as the correlation of data. Aggregating the data in the group leader enables DPDS to accurately calibrate the noise to the data properties. In contrast, the existing works only consider the sensory data of a single user to add noise. The added noise to all users is then aggregated in the server, which results in degrading the utility.
The stability of our proposed system is evaluated in Figure 7 . For each privacy budget, the utility of algorithms is computed over a range of time intervals. Each value in $\mathrm{x}$ axis depicts a time interval, that is, the number of consecutive timestamps that the algorithm has collected data from IoT devices and sent to the server. Note that existing algorithms do not consider the accumulation of privacy loss in the server. This limitation restricts the algorithms only to finite streams with a specified length. To highlight the effect of this limitation, we adjust the total privacy budget in these algorithms to the budget usage in DPDS. For example, assume the time interval is 60 seconds, and DPDS has used an $\epsilon$ budget during this time. This means from the total six timestamps in this interval (the sampling rate is 10 seconds) DPDS has spent $\epsilon$ only in one of them and then other timestamps are filtered by the algorithm. In such a case, for the other algorithms, we assign $\frac{\epsilon}{6}$ for each timestamp to compute the output. As the results show, DPDS accurately captures significant changes 
in data, and our filtering strategy enables DPDS to be stable over time. In contrast, the competing algorithms suffer from the noise effect and their utility is not stable over the time.

\section{CONCLUSION}

We proposed a differentially private data streaming system for ensuring privacy in the IoT layer of the edge computing architecture when the transportation infrastructure (edge controller and server) is not a trusted entity. The IoT devices that are subscribed to a controller select a group leader to collect and perturb the sensory data. The group leader forms a matrix of the sensory data, compress it, and then sends the compressed data to the edge controller. We proposed compression and thresholding techniques which use data properties to reduce the scale of perturbation, and hence, output a high utility data. Also, we propose a filtering strategy that enables our mechanism to maintain a high utility as well as a high level of privacy over time. Our extensive experiments show that considering data properties in calibrating the noise significantly improves the utility when the noise is added in the IoT layer. We described our proposed system with an application to ITS. However, our proposed perturbation mechanism can be applied to any other similar dynamical system where data is highly correlated in the IoT layer, and the IoT devices can communicate with one another, can form groups, and can change their edge subscription. As a future direction, we aim to analyse the trade-offs between the efficiency of system and the accuracy of output to maximize the utility and minimize the adverse impacts of perturbations on the output utility. We will also aim to minimize the computation complexity by optimizing the compression strategy used for reducing the scale of noise.

\section{REFERENCES}

[1] N. Malik, D. Puthal, and P. Nanda, "An overview of security challenges in vehicular ad-hoc networks," in 2017 International Conference on Information Technology (ICIT). IEEE, 2017, pp. 208-213.

[2] A. Jolfaei, K. Kant, and H. Shafei, "Secure data streaming to untrusted road side units in intelligent transportation system," in 18th IEEE International Conference on Trust, Security and Privacy in Computing and Communications, 2019.

[3] S. Garg, K. Kaur, N. Kumar, and J. J. P. C. Rodrigues, "Hybrid deeplearning-based anomaly detection scheme for suspicious flow detection in SDN: A social multimedia perspective," IEEE Transactions on Multimedia, vol. 21, no. 3, pp. 566-578, 2019.

[4] S. Garg, K. Kaur, G. Kaddoum, S. H. Ahmed, and D. N. K. Jayakody, "Sdn based secure and privacy-preserving scheme for vehicular networks: A 5g perspective," IEEE Transactions on Vehicular Technology, 2019.

[5] F. Farivar, M. Sayad, A. Jolfaei, and M. Alazab, "Artificial intelligence for detection, estimation, and compensation of malicious attacks in nonlinear cyber physical systems and industrial iot," IEEE Transactions on Industrial Informatics, 2019.

[6] N. Malik, P. Nanda, A. Arora, X. He, and D. Puthal, "Blockchain based secured identity authentication and expeditious revocation framework for vehicular networks," in 2018 17th IEEE International Conference On Trust, Security And Privacy In Computing And Communications/12th IEEE International Conference On Big Data Science And Engineering (TrustCom/BigDataSE). IEEE, 2018, pp. 674-679.

[7] S. Garg, K. Kaur, S. H. Ahmed, A. Bradai, G. Kaddoum, and M. Atiquzzaman, "Mobqos: Mobility-aware and qos-driven sdn framework for autonomous vehicles," IEEE Wireless Communications, vol. 26, no. 4, pp. 12-20, 2019.
[8] M. Usman, M. A. Jan, and D. Puthal, "Paal: A framework based on authentication, aggregation and local differential privacy for internet of multimedia things," IEEE Internet of Things Journal, 2019.

[9] C. Dwork, A. Roth et al., "The algorithmic foundations of differential privacy," Foundations and Trends in Theoretical Computer Science, vol. 9, no. 3-4, pp. 211-407, 2014.

[10] S. Ghane, L. Kulik, and K. Ramamohanarao, "TGM: A generative mechanism for publishing trajectories with differential privacy," IEEE Internet of Things Journal, 2019.

[11] S. Ghane, L. Kulik, and K. Ramamohanarao, "Publishing spatial histograms under differential privacy," in 30th ACM International Conference on Scientific and Statistical Database Management (SSDBM), 2018, pp. 14:1-14:12.

[12] L. Fan and L. Xiong, "Real-time aggregate monitoring with differential privacy," in 21st ACM international conference on Information and knowledge management, 2012, pp. 2169-2173.

[13] Q. Wang, Y. Zhang, X. Lu, Z. Wang, Z. Qin, and K. Ren, "Rescuedp: Real-time spatio-temporal crowd-sourced data publishing with differential privacy," in 35th Annual IEEE Conference on Computer Communications (INFOCOM), 2016, pp. 1-9.

[14] A. Jolfaei and K. Kant, "Privacy and security of connected vehicles in intelligent transportation system," in 49th IEEE/IFIP International Conference on Dependable Systems and Networks, 2019, pp. 9-10.

[15] S. Ghane, A. Jolfaei, L. Kulik, and K. Ramamohanarao, "Differentially private streaming to untrusted edge servers in intelligent transportation system," in 18th IEEE International Conference On Trust, Security And Privacy In Computing And Communications, 2019.

[16] J. C. Duchi, M. I. Jordan, and M. J. Wainwright, "Minimax optimal procedures for locally private estimation," Journal of the American Statistical Association, vol. 113, no. 521, pp. 182-201, 2018.

[17] N. Wang, X. Xiao, Y. Yang, J. Zhao, S. C. Hui, H. Shin, J. Shin, and G. Yu, "Collecting and analyzing multidimensional data with local differential privacy," in 35th IEEE International Conference on Data Engineering, 2019.

[18] S. Garg, A. Singh, K. Kaur, G. S. Aujla, S. Batra, N. Kumar, and M. S. Obaidat, "Edge computing-based security framework for big data analytics in vanets," IEEE Network, vol. 33, no. 2, pp. 72-81, 2019.

[19] M. Joseph, A. Roth, J. Ullman, and B. Waggoner, "Local differential privacy for evolving data," in Advances in Neural Information Processing Systems, 2018, pp. 2381-2390.

[20] G. Kellaris, S. Papadopoulos, X. Xiao, and D. Papadias, "Differentially private event sequences over infinite streams," VLDB Endowment, vol. 7, no. 12 , pp. $1155-1166,2014$

[21] O. E. Agboje, O. B. Idowu-Bismark, and A. E. Ibhaze, "Comparative analysis of fast fourier transform and discrete wavelet transform based mimo-ofdm," International Journal of Communication Antenna and Propagation, vol. 7, no. 2, pp. 168-75, 2017.

[22] N. Umezu, K. Asai, and M. Inui, "Wavelet transform data compression with an error level guarantee for z-map models," International Journal of Automation Technology, vol. 10, no. 2, pp. 201-208, 2016.

[23] J. Nowaková, M. Prilepok, and V. Snášel, "Medical image retrieval using vector quantization and fuzzy s-tree," Journal of medical systems, vol. 41 , no. 2 , p. 18,2017

[24] H. Liao, M. K. Mandal, and B. F. Cockburn, "Efficient architectures for 1-d and 2-d lifting-based wavelet transforms," IEEE Transactions on Signal Processing, vol. 52, no. 5, pp. 1315-1326, 2004.

[25] S. Bassoy, H. Farooq, M. A. Imran, and A. Imran, "Coordinated multipoint clustering schemes: A survey," IEEE Communications Surveys \& Tutorials, vol. 19, no. 2, pp. 743-764, 2017.

[26] A. Jolfaei and K. Kant, "A lightweight integrity protection scheme for low latency smart grid applications," Computers \& Security, vol. 86, pp. 471-483, 2019.

[27] A. Jolfaei, X.-W. Wu, and V. Muthukkumarasamy, "A secure lightweight texture encryption scheme," in Image and Video Technology, 2015, pp. 344-356.

[28] S. Uppoor and M. Fiore, "Large-scale urban vehicular mobility for networking research," in 2011 IEEE Vehicular Networking Conference (VNC), 2011, pp. 62-69.

[29] A. Jolfaei, A. Vizandan, and A. Mirghadri, "Image encryption using hc-128 and hc-256 stream ciphers," International Journal of Electronic Security and Digital Forensics, vol. 4, no. 1, pp. 19-42, 2012. 